\title{
The Design of Smart Meter with Power Quality Monitoring
}

\author{
Shihui Yang ${ }^{1, \mathrm{a}}$, Fangfang Duan ${ }^{1, \mathrm{~b}}, \mathrm{Ke} \mathrm{Li}^{2, \mathrm{c}}$, Bin $\mathrm{Li}^{1, \mathrm{~d}}$ \\ ${ }^{1}$ State Grid Henan Electric Power Company Luoyang Power Supply Company, Luoyang 471000, \\ China \\ ${ }^{2}$ State Grid Boai Power Supply Company, Jiaozuo 454450, China \\ a luoyangdidiao@sina.com, b1015903240@qq.com
}

Keywords: Smart Grid, Smart Meter, Power Quality Monitoring.

\begin{abstract}
In recent years, improving power quality has become an urgent need to protect the safe of power system, stable operation and meet user's requirements. The existing power quality monitoring system, mainly to get the data through special acquisition equipment of power quality, it is not for real-time monitoring of each user's power consumption. In order to meet the power quality requirements of smart grid, we present a design scheme of power quality information from smart meter. It can achieve the sharing of resources within the grid, good real-time and wider range of monitoring. In order to meet the smart grid power quality requirements, we proposed a design scheme to obtain power quality information sharing can be achieved within the grid resources from strong real-time monitoring a wider range of smart meters.
\end{abstract}

\section{Introduction}

With the rapid development of communication technology, information processing technology and automation technology, in promoting a low-carbon economy and green energy-saving ideas for how to further improve grid efficiency and to address environmental challenges and improve reliability and power quality, improve electricity customer service and giving more and more attention. Therefore, the smart grid is an electricity inevitable development today is the development direction of the power system. Smart Grid is based on the traditional power grid, the integration of computers, communications, sensing and other technologies in one of the new grid, with digitization, automation, information technology and basic interactive features. It is mainly through the terminal sensor between the user, the company formed between the user and the grid network of interactive real-time access, real-time, high speed, bidirectional read data, improve overall grid overall.

Faced with the current status of the lack of electrical energy, smart grid will become the first choice for sustainable social and economic development. Energy conservation, we must improve the ability to control the grid, not only to reduce the losses in the transport and distribution sectors, but also the flexibility to receive the renewable energy network; operational aspects of security and stability, strengthening the power grid self-protection capability to withstand natural disasters, to exclude outside interference, but also have intellectual ability to repair itself, improve grid efficiency of equipment, improve power quality; the user side, the installation of smart meters to achieve information exchange, to meet each user's electricity demand.

Smart meters as one of the important smart grid infrastructure, is a microprocessor applications and network communication technology as the core, by the measurement unit, a data processing unit, a communication unit and other components of intelligent instruments, with energy measurement, information storage and processing, real-time monitoring, automatic control, information exchange and other functions. Accelerate its intelligent application and improve functional, for a grid based on information technology, automation, interactive play an important supporting role. Meanwhile, in the framework of the smart grid, a smart meter requires the detection of information and collectors, data is passed between the grid and the users of the bridge. Smart meters as client smart grid, the energy data collected by it to implement the data sent to the grid in various smart devices, smart grid data receiving terminal and power parameters to adjust and 
improve the quality of electricity users.

\section{The Functions of Smart Meter}

Unlike the smart meter multi-function table power meter, because it itself contains more powerful microcontroller (MCU), so it's like a mini computers, automation and intelligent features richer, more powerful, usually it will have some of the following major functions.

(1) Two-way metering function is a basic function of smart meters, including voltage, current, frequency, active power, reactive power, power factor, apparent power and energy metering in. The measurement voltage and current requirements to provide sufficient measurement data points, historical data for the realization of advanced applications of statistical analysis.

(2) Supports floating electricity smart meter internal built real time clock, and the time parameter to the meter, it is possible to automatically switch period, interval measurement and storage of various types of energy and consumption data based on pre-set time, and support points when electricity or real-time pricing and demand-side management, to meet the real-time energy metering under tariff.

(3) Two-way communication is one-way communication function was significantly different from the traditional characteristics of the smart meter energy meter, the smart meter built-in communication module, two-way exchange of information through communication networks and data centers. Thus, the intelligent smart grid substation in addition to accept electricity smart meters send out information, you can also send out information to the smart meter regulation. Intelligent substation communication between smart meters and the remote can take many forms, such as power line carrier communications, wireless network communications, communications network communications and other means of private network communication (such as cable TV networks, etc.). The future development of triple play will bring greater convenience to smart metering communication.

(4) Control and management control and management functions include freeze electricity information data, storage, and loading of records of events, the state quantity measurement and alarm. Smart meter control and management functions can be well applied to intelligent home appliances to use its powerful interface capabilities and remote communications capabilities to achieve the set parameters such as air conditioning, water heaters, washing machines and other home appliances remote switch control based on the spot price.

\section{Smart Meter System Design}

Smart grid smart meters in support of electricity load management, distributed power grid, aspects of power system operation scheduling, power quality monitoring and power market transactions, etc. There are many requirements. Existing smart meters is the main achievement of power measurement, the price ladder, electricity billing functions. In order to further improve the capabilities of smart meters in power quality monitoring, this paper proposes a new smart meter design program, which can be achieved in addition to the above features, but also capable of power quality monitoring.

Smart meters plus a dedicated single-chip energy metering chip design, the power parameters and energy measurements are done directly by the ATT7022B chip, can be attributed to the voltage and current, AC signal inputted AC sampling, and then a series of linear transformation, apply some algorithm to calculate the RMS voltage and current, power, power factor, frequency and power and so on. Based on the above functional requirements, combined with special energy metering chip ATT7022B, it introduced the basic parameters of electric power and power quality parameters, and gives the corresponding calculation method provides a theoretical basis for the software design.

RMS voltage is defined as the rms value of periodically changing voltage signal. By squaring the sample values of voltage, extraction, filtering and a series of operation to obtain the phase voltage rms. RMS current rms value is defined as the periodic change in the current signal of the current sample value by square, extraction, filtering sequence of operations to obtain the phase current 
RMS. Active power is required to maintain the normal operation of electrical equipment electric power, defined as the average of the instantaneous power over a cycle. Because energy storage elements mean instantaneous power of one cycle 0 , so the actual element that is active on the circuit resistance energy size. After measuring principle by the DC component of the current and voltage go after multiplication, addition, digital filtering and a series of digital signal processing to give each phase active power. There are function defined as the amount of electromagnetic energy that can be converted into other forms of energy, electrical energy, through instantaneous active power integrating to the time available. Reactive power is unable to work outside the grid of power, but electricity grid equipment must require reactive power to run, so the reactive power to maintain grid work is one of the essential aspects of safe operation of power grids, it has a crucial role. The reactive power measurement is similar to the active, just after the phase-shifted by 90 degrees voltage signal used. Mainly due to the measurement bandwidth digital phase bandwidth limiting filter amplitude-frequency characteristic choice of 1 , all frequency components within the frequency range will be -90 o phase shift linear filter, can achieve high measurement accuracy. ATT7022B direct output voltage and frequency parameters, and can automatically select A, B, C in any one of the three-phase voltage measuring voltage frequency measurement benchmarks. The standard grid frequency is $50 \mathrm{~Hz}$; ATT7022B can be measured in the range of $10 \mathrm{~Hz} \sim 500 \mathrm{~Hz}$, high measurement accuracy. Power factor means that the load on the active power and apparent power ratio, one of the main purpose of measurement is to calculate the reactive power factor. The power factor of the power system as an important indicator of its size reflects the level of quality of the power grid, it is focused on the power sector regulator grid reference data.

\section{Smart Meter System Function Design}

Data acquisition module is divided into three parts, one read from the internal register ATT7022B voltage rms, current rms, active or reactive power, active or reactive power, apparent power, power factor, frequency and harmonics related parameters; two is the data conversion, the voltage, current transformer access, ATT70222B internal A / D converter process, so want to read register values converted again calculated to obtain the actual measured value; Third power quality is estimated by measuring the actual values into the appropriate formula to give power quality.

Whether it is sending or receiving data, it must be carried out in case the bus is not busy. When the bus is not busy, the data line (SDA) and a clock line (SCL) is high. When SCL is high, SDA called the emergence of a falling edge of the start signal (S); when SCL is high, SDA emergence of a rising edge, called the stop signal (P). When transferring data, shown in Figure 4-8, each clock pulse transmits a data bit, byte transmitted first high, then low byte transfer, and the data bits are transmitted by the SDA line high, low power Ping said that the data on the SDA line remains stable at a high level when the clock pulse, otherwise the data on the SDA line will be the above mentioned control signal.

The design uses a built-LCD T6963C controller display module, a set of separate instruction system. General initialization module by pin setting is completed; the instruction focused on setting display functions. Each instruction can take one, two parameters or no parameters. Each instruction execution are first fed parameters (no arguments, do not send), and then into the instruction code.

\section{Summary}

With the rapid development of smart grid, smart meter applications will continue to expand. At present, the smart meter cannot fully meet the demand for power management, in order to more effectively monitor user power quality, and timely handling emergencies, improve power management efficiency, smart meters need to continue to add new features, higher technical indicators. Thus, smart meters will be better able to adapt to the requirements of the smart grid. 


\section{Reference}

[1] Chang T Y, Wang Y, Sun H J. The Design of Smart Meter with Power Quality Monitoring[J]. Electrical Measurement \& Instrumentation, 2012.

[2]Hoglund J, Ilic D, Karnouskos S, et al. Using a 6LoWPAN smart meter mesh network for event-driven monitoring of power quality[C]// Smart Grid Communications (SmartGridComm), 2012 IEEE Third International Conference on. IEEE, 2012:448 - 453.

[3]Koponen P, Seesvuori R, Bostman R. Adding power quality monitoring to a smart kWh meter[J]. Power Engineering Journal, 1996, 10(4):159 - 163.

[4]Wang Q. Analysis and Application of Obtaining Power Quality Data from Smart Meter[J]. Power \& Energy, 2012.

[5]Benyoucef D, Klein P, Bier T. Smart Meter with non-intrusive load monitoring for use in Smart Homes[C]// Energy Conference and Exhibition (EnergyCon), 2010 IEEE International. IEEE, 2010:96 - 101.

[6]Tse N C F, Chan J Y C, Lau W H, et al. Real-Time Power-Quality Monitoring With Hybrid Sinusoidal and Lifting Wavelet Compression Algorithm[J]. IEEE Transactions on Power Delivery, 2012, 27(4):1718 - 1726. 